\title{
Intermédialités
}

Histoire et théorie des arts, des lettres et des techniques

Intermediality

History and Theory of the Arts, Literature and Technologies

\section{Reconstituer Waterloo dans les années 1960 : l'histoire selon Marcel Broodthaers et Norbert Brassinne}

\section{Nicolas Fourgeaud}

Numéro 28-29, automne 2016, printemps 2017

refaire

redoing

URI : https://id.erudit.org/iderudit/1041087ar

DOI : https://doi.org/10.7202/1041087ar

Aller au sommaire du numéro

Éditeur(s)

Revue intermédialités (Presses de l’Université de Montréal)

ISSN

1920-3136 (numérique)

Découvrir la revue

Citer cet article

Fourgeaud, N. (2016). Reconstituer Waterloo dans les années 1960 : l'histoire selon Marcel Broodthaers et Norbert Brassinne. Intermédialités / Intermediality, (28-29). https://doi.org/10.7202/1041087ar
Résumé de l'article

Avec cet article, on souhaiterait faire le portrait (contextuel, institutionnel, médiatique) de deux acteurs fondamentalement différents, mais qui font de la reconstitution historique un enjeu central de leurs pratiques dès la fin des années 1960 et la rapportent, dans un cas comme dans l'autre, à un même lieu, le champ de bataille de Waterloo, en Belgique francophone : l'artiste bruxellois Marcel Broodthaers (1924-1976) et le commerçant/reconstituteur wallon Norbert Brassine (1907-1988). En plus d'analyser quel rapport à l'histoire et à la reconstitution développent ces deux non-historiens très distincts à travers leurs actions, leurs productions ou leurs apparitions médiatiques, cet article vise aussi à revenir sur une période où la reconstitution historique en était à ses balbutiements à Waterloo, la fin des années 1960. Ce double portrait se propose donc comme une archéologie très localisée des usages et représentations de la reconstitution historique. 


\title{
Reconstituer Waterloo dans les années 1960 : l'histoire selon Marcel Broodthaers et Norbert Brassinne
}

\author{
Nicolas Fourgeaud
}

ourquoi je me suis déplacé à l'époque jusqu'à Waterloo, je ne me le rappelle plus $^{1} \gg, s^{\prime}$ interroge un personnage du romancier allemand W. G. Sebald, dans une Europe fin de $20^{e}$ siècle infiniment présentiste ${ }^{2}$. Dans le village commercial quasiment vide qui jouxte le champ de bataille belge surgissent bientôt devant le narrateur ici des hommes-automates exécutant une marche en uniforme napoléonien, là un panorama poussiéreux qui donne l'occasion à la voix du roman de ruminer sur «la perspective faussée » de toute tentative de représentation de l'histoire. Expression d'une transformation de la geste napoléonienne en matière à actions commerciales, cette scène nous renvoie aussi, en un sens, à une des fondations de la reconstitution historique contemporaine : la «tradition inventée ». En 1983, alors qu'allait commencer en France une vaste entreprise éditoriale visant à lutter contre l'oubli des histoires, lieux et symboles fondateurs de l'identité nationale française ${ }^{3}$, un collectif d'historiens britanniques mené par Eric Hobsbawm et Terence Ranger proposait de manière plus corrosive une série de travaux décrivant comment, à la fin du $18^{\mathrm{e}}$ siècle, les élites dominées de l'Écosse ou du pays de Galles, mais aussi la monarchie britannique au $19^{\mathrm{e}}$ siècle, s'étaient inventées en tant que nations en s'appropriant ou en créant ex nibilo traditions populaires, épopées de la plus grande antiquité en langues vernaculaires, costumes nationaux ou rituels ${ }^{4} . .$. Le moment

${ }^{I}$ W. G. Sebald, Les anneaux de Saturne [1995], Arles, Actes Sud, 1999, p. I47.

${ }^{2}$ Sur le présentisme comme régime d'historicité dominant aujourd'hui, voir François Hartog, Régimes d'bistoricité [2003], Paris, Éditions du Seuil, 2012.

3 Il s'agit bien sûr des Lieux de mémoire, entreprise collective dirigée par l'historien Pierre Nora, dont le tome I, La République, est publié en 1984 aux éditions Gallimard.

4 Eric Hobsbawm et Terence Ranger (dir.), L'invention de la tradition [1983], Paris, Éditions Amsterdam, 2012. 
romantique voit la naissance de ce mouvement d'invention des identités nationales, qui va essaimer d'abord en Europe et sur le continent américain. Par l'histoire, par la langue, par des gestes, les porteurs des revendications nationales visent à donner une légitimité culturelle et politique à leur ambition de rassembler une communauté au sein d'un espace politique autonome. Si la nation est un projet politique, elle est aussi un projet médiatique. Ainsi, paysages, arts visuels, musique, sport et timbre-poste sont mis à contribution dans ce grand mouvement de création des identités nationales, tout comme les expositions universelles, les musées d'art, puis, à la fin du $19^{\mathrm{e}}$ siècle, d'ethnographie avec leurs dioramas et leurs visites guidées en costume folklorique. Porteuses de l'idéologie du progrès, du capitalisme et de l'industrialisation, les nations se jettent dans l'avenir en contestant leurs propres fondements par le recours à un archaïsme délibéré, ainsi que le souligne Anne-Marie Thiesses.

La reconstitution historique ${ }^{6}$ telle qu'elle prend son essor en Europe et aux États-Unis au courant des années I970, et surtout 1980, est l'héritière populaire de ce mouvement de fond romantique : elle est, même à l'ère de la globalisation et malgré un rapport fort à l'ici et maintenant, fille de l'État-nation et des «imaginaires nationaux $\gg^{7}$. Proche de ses ancêtres «inventés » du $19^{\mathrm{e}}$ siècle, elle est teintée d'agendas mémoriels, politiques, communicationnels et commerciaux tout à la fois. Nous nous proposons dans cet article de revenir sur la seconde moitié des années 1960, en Europe de l'Ouest, alors que la reconstitution commence tout juste à balbutier et qu'elle n'est pas encore devenue cette pratique somatique et mémorielle essentielle à notre contemporanéité. À cette époque, les Trente Glorieuses ne sont pas encore achevées, et, dans cette phase d'après-guerre, les cultures nationales ont rencontré la culture et le tourisme de masse. Aux nationalismes étatiques autoritaires et expansionnistes des années 1930, l’Europe de l’Ouest préfère désormais les revendications identitaires régionalistes, particulièrement en France (Occitanie, Bretagne) et en Belgique (Wallonie, Flandre). Les lieux de mémoire ne sont pas encore

5 Voir Anne-Marie Thiesse, La création des identités nationales, Europe I8 siècle-2o siècle, Paris, Éditions du Seuil, 1999.

${ }^{6} \mathrm{Par} \ll$ reconstitution historique », nous entendons ici la recréation ou la réinvention d'événements historiques (batailles, voyages) à l' « échelle I ». Nous ne prenons pas en compte les musées d'histoire vivante (Living History Museums), dont l'histoire remonte au début du $20^{\mathrm{e}}$ siècle.

7 Voir Benedict Anderson, L'imaginaire national. Réflexions sur l'origine et l'essor $d u$ nationalisme [1983], Paris, Éditions La Découverte, 1996. 
la matière préférée des historiens, et les interrogations sur la mémoire collective ou le rapport au passé des sociétés présentes ne sont pas encore légion - l'archive vient tout juste de gagner son usage singulier grâce à Michel Foucault ${ }^{8}$, et le tumulte des mouvements de décolonisation se fait encore entendre. C'est sur ce fond rapidement esquissé qu'on souhaiterait modestement faire le portrait (contextuel, institutionnel, médiatique) de deux acteurs fondamentalement différents, mais qui font de la reconstitution historique un enjeu central de leurs pratiques et la rapportent, dans un cas comme dans l'autre, à un même lieu phare du romantisme, le champ de bataille de Waterloo en Belgique francophone.

Dès la fin des années 1960, l'artiste belge Marcel Broodthaers (1924-1976) commence à questionner les formes d'écriture de l'histoire, et la reconstitution, sous diverses formes, va dès lors s'imposer à lui comme un enjeu important. Le champ de bataille de Waterloo apparaît rapidement dans son œuvre comme une allégorie de cet enjeu. À cette période, histoire vernaculaire et tradition inventée se mélangent joyeusement à Waterloo, et on peut y croiser des proto-reconstitutions historiques et divers rituels réalisés par un commerçant local nommé Norbert Brassinne (19071988), un passionné d'histoire napoléonienne. Nous proposerons ici un double portrait, celui du reconstituteur, mal connu encore, emboîté dans celui dédié à l'artiste. S'y dessineront deux rapports spécifiques à l'histoire, l'un se formulant dans des œuvres émanant du champ de l'art avancé, l'autre dans des actions, au statut peu évident, relayées par différents médias, et notamment la télévision où nous irons puiser de nombreux documents. Tout au long de ce texte, nous nous emploierons à montrer que Broodthaers n'a pas été inattentif au revival du folklore dans la Belgique d'alors, ni aux démonstrations de son proche voisin de Waterloo, alors même que les pratiques de reconstitution historique sont un phénomène plus que mineur dans cette Europe des années 1960. Plus généralement, nous voudrions soutenir que Broodthaers conçoit Waterloo comme le lieu de reproduction de conceptions de l'Histoire et de la mémoire venues $\mathrm{du}_{\mathrm{i}} \mathrm{9}^{\mathrm{e}}$ siècle européen, et en particulier du moment romantique. Norbert Brassinne, lui, jouera le rôle d'indicateur informel du développement d'un certain rapport nostalgique à l'Histoire dans les pays industrialisés des années 1960. Plutôt que de l'envisager comme l'inventeur d'un genre, nous l'approcherons plus simplement comme un acteur précoce d'une pratique performantielle importante dans les formes contemporaines $\mathrm{du}$

\footnotetext{
${ }^{8}$ Michel Foucault, L'archéologie du savoir [1969], Paris, Gallimard, 2005.
} 
nationalisme populaire9 et du commerce de l'Histoire. À travers son portrait, ses gestes d'appropriation des grands récits et des formes (monumentales ou rituelles) de la mémoire officielle, le lecteur verra peut-être s'esquisser les grandes caractéristiques des sociétés du patrimoine des années 1980 : fièvre de la mémoire, multiplication des acteurs et producteurs d'Histoire locaux sans point d'ancrage académique, développement de nouvelles formes d'appropriation et de mise en narration du patrimoine (la reconstitution étant un outil essentiel ici), et, surtout, volonté affirmée chez de nombreux protagonistes d'écrire une Histoire locale à soi qui ne soit pas antagonique aux méta-récits nationaux - quitte à s'arranger avec la réalitée ${ }^{10}$.

\section{LE I $9^{E}$ SIÈCLE DE MARCEL BROODTHAERS}

« Ensuite, un voyage itinérant de mon musée, spécialement le "Département des Aigles", à Waterloo dans le cadre doré du bicentenaire de la naissance de Napoléon. Succès de foule, mon cher, j'en ai rapporté un petit film à titre de souvenir personnel que j'ai moi-même tourné avec des soldats ${ }^{\text {II }}$. »

Dans les années qui suivent la Seconde Guerre mondiale, Broodthaers, né en 1924, goûte au surréalisme révolutionnaire belge, à la bohème désargentée, et fréquente les rivages très confidentiels de la poésie, pour finir par quitter un milieu par trop cénaculaire en 1964. Il se déclare alors officiellement « artiste » et intègre un nouveau réseau institutionnel et une nouvelle économie. Influencé par l'art américain contemporain alors largement diffusé en France et en Allemagne, il développe

9 Pour un traitement de la dimension performantielle du nationalisme populaire contemporain, voir notamment Tim Edensor, National Identity, Popular Culture and Everyday Life, Oxford, New York, Berg, 2002, p.69-I02. Pour une approche de la reconstitution historique, voir Brad West, « Historical Re-enacting and Affective Authority: Performing the American Civil War », Annals of Leisure Research, n², 2014, p. 16I-179.

ro Voir le texte fondateur de Pierre Nora, «L'ère de la commémoration », dans Pierre Nora (dir.), Les Lieux de mémoire [1992], Paris, Gallimard, 1997, p. 4699-4706. Pour des travaux récents sur la question, voir les contributions du Laboratoire d'anthropologie et d'histoire de l'institution de la culture (CNRS/EHESS), créé par l'anthropologue Daniel Fabre en 20or. Pour l'ensemble des points évoqués dans la dernière partie de cette introduction, voir notamment les volumes collectifs publiés aux éditions de la Maison des sciences de l'homme : Alban Bensa et Daniel Fabre (dir.), Une histoire à soi. Figurations du passé et localités, Paris, 200I; Daniel Fabre (dir.), Émotions patrimoniales, Paris, 2013; Daniel Fabre (dir.), Domestiquer l'histoire. Ethnologie des monuments historiques, Paris, 2016.

"I Marcel Broodthaers, Lettre à Jacques Charlier, Bruxelles, le 21 juillet 1969, cité par Hans Theys, Marcel Broodthaers: un voyage à Waterloo, Gent, MERZ, 20oI, non paginé. 
pendant quelques années un art de société, une sorte de pop art nourri aux mythologies de la culture vernaculaire belge, avec ici et là quelques références directes et moqueuses aux symboles nationaux et à la décolonisation au Congo belge ${ }^{12}$. À partir de 1968, alors que son travail s'est déjà résolument infléchi vers le rapport imagetexte (1967), Broodthaers affirme un tropisme $19^{\mathrm{e}}$ siècle, présent depuis des années déjà dans sa culture personnelle, ses films ou encore ses articles pour la presse. Complètement à rebours des tendances anti-histoire dominantes à l'époque (dans l'art conceptuel et l'art minimal), il installe des fragments de culture visuelle et littéraire romantique et post-romantique française au cœur de ses travaux. Ces morceaux de Grandville, Dumas ou Mallarmé, loin d'être de simples coquetteries érudites, pointent vers le conflit entre art autonome et « art industriel », comme on disait au $19^{\mathrm{e}}$ siècle, dont les années I830, en France, ont sonné la naissance. La plupart des auteurs dont se saisit l'artiste belge ont en effet thématisé la question du statut de l'art à l'ère industrielle. Broodthaers, en jouant avec l'histoire sociale du champ littéraire (l'appropriation étant en plus une opération clé du commerce des lettres pendant la période romantique, notamment en Belgique), entend commenter de manière indirecte les conditions de production et de diffusion de l'art contemporain de son temps : émergence en Europe d'un nouveau réseau de galeries (en Allemagne, aux Pays-Bas, en Italie et en Belgique ${ }^{\mathrm{I3}}$ ), développement de foires d'art contemporain (en Allemagne et en Suisse) en réponse à la montée en puissance des États-Unis sur le marché de l'art ${ }^{14}$, premières injections significatives de capitaux d'entreprises dans le monde de l'art avancé (voir Quand les attitudes deviennent formes, $\left.1969^{\mathrm{IS}}\right) \ldots$.. Le nouveau système de l'art vient donc se refléter dans les figures $\mathrm{du}$ lointain $19^{e}$ siècle. L'histoire n'est pas en reste dans le devenir-marchandise de l'art au $19^{\mathrm{e}}$ siècle. Elle est un matériau de prédilection et une ressource commerciale

${ }^{12}$ Sur les enjeux liés à l'identité nationale dans cette période du travail de Broodthaers, on signalera les réflexions de Rachel Haidu, chapitre I, «Identity and Wit », The Absence of Work: Marcel Broodthaers 1964-1976, Cambridge, Londres, MIT Press, 2013, p. I-45.

${ }^{13}$ Broodthaers travailla avec une galerie phare de ce nouveau réseau de marchands d'art, la Wide White Space Gallery à Anvers. Sur ce réseau, voir Sophie Richard, Unconcealed. The International Network of Conceptual Artists 1967-1977. Dealers, Exbibitions and Public Collections, Londres, Ridinghouse, 2009.

${ }^{14}$ Sur la géopolitique de l'art occidental dans les années 1960 et 1970, voir Catherine Dossin, The Rise and Fall of American Art, 1940's-1980's: a Geopolitics of Western Art Worlds, Burlington, Ashgate Publishing Co, 2015.

is La branche européenne du cigarettier Philip Morris sponsorisa l'exposition d'Harald Szeeman. 
fondamentale pour le roman romantique. Ce n'est donc pas un hasard si, en 1969, Broodthaers superpose son nom à celui de Dumas sur une édition de poche de Vingt ans après, une fiction exemplaire d'un certain usage spectaculaire de l'histoire.

Si l'artiste dresse une certaine généalogie de la marchandisation de l'art et de l'histoire, il ne fait pas pour autant main basse sur les méthodologies des sciences humaines et sociales. Broodthaers, on le sait, préfère prendre le parti de l'allégorie - un trope à la croisée du visuel et du discursif, un hybride de texte et d'image à la fois. L'allégorie broodthaersienne, malgré son opacité, est nettement historienne : elle résulte d'un processus d'addition et de cumul voire de mise en collection visant à proposer des mises en relation et à dessiner ainsi une certaine histoire. L'artiste joue à mettre en écho des historicités distinctes et dessine un certain « ordre du temps », pour reprendre l'expression de François Hartog ${ }^{16}$. Et ici, la figure du musée est évidemment fondamentale. Le moment $1^{\mathrm{e}}$ siècle de la pratique de Broodthaers est justement inauguré par son Musée d'art moderne, Département des Aigles, Section $\mathrm{XIX}^{\circ}$ siècle, le 28 septembre 1968 - une allégorie de l'histoire et de ses modes d'ordonnancement institutionnel à l'ère moderne. Ce musée en milieu domestique est composé des signes de l'institution (caisses d'œuvres d'art, discours d'inauguration, papier à en-tête) et d'œuvres d'art « remédiées » (cartes postales scotchées au mur reproduisant des peintures $\mathrm{du} 19^{\mathrm{e}}$ siècle, projections de diapositives faisant la part belle aux caricaturistes français $\mathrm{du}_{1} 9^{\mathrm{e}}$ siècle). Jeu de signes, le Musée des Aigles se veut un moyen d'interroger la fonction de l'institution muséale dans une séquence historique où les musées tissent de plus en plus de liens avec le champ de l'art contemporain et ses instances de vente et de distribution ${ }^{17}$. C'est fort de ce musée installé dans son appartement que Broodthaers va faire sa première incursion artistique à Waterloo. Nous sommes en 1969, année du bicentenaire de la naissance de Napoléon et du départ du général de Gaulle, lorsque l'artiste belge tourne un court film muet, en $16 \mathrm{~mm}, U n$ voyage à Waterloo (Napoléon $1769-1969)^{18}$. Après un carton

${ }^{16}$ Sur Broodthaers et l'allégorie, voir notamment Benjamin Buchloh, «Allegories of the Avant-Garde », Artforum, mai 1980, p. 52-59. Sur l'expression «ordre du temps », voir Hartog, 20I2.

${ }_{17}$ Voir Marcel Broodthaers, texte de 1968 introduisant le film Une discussion inaugurale, cité par Manuel J. Borja-Villel, Christophe Cherix et al., Marcel Broodthaers: a Retrospective, New York, MoMA, 2016, p. I78.

${ }^{18}$ Il existe au moins trois versions du film. Voir la notice « Un voyage à Waterloo », dans Manuel J. Borja-Villel, Michael Compton et Maria Gilissen(dir.), Marcel Broodthaers: cinéma, Barcelone, Fundacio Antoni Tàpies, 1997, p. 82. La version sur laquelle nous avons travaillé comporte quelques différences mineures par rapport à celles décrites dans ce catalogue. 
indiquant « Fragile », Broodthaers, équipé d'un gros nez comme on peut en utiliser au théâtre, déclame quelques phrases silencieuses sur le parc de stationnement du village touristique du champ de bataille. Il s'assoit ensuite sur une caisse, à côté d'un canon, non loin d'un autocar touristique. Puis, une courte dérive à Bruxelles, dans un périmètre très restreint, nous conduit de la rue Brederode (juste derrière le Palais royal de Bruxelles) au Musée de la Dynastie, puis jusqu’à la rue de la Pépinière, où Broodthaers est domicilié. Se succèdent une série d'inscriptions institutionnelles : un panneau de signalisation routière, la plaque signalant le Musée de la Dynastie, le mot «MUSÉE », sur une vitre, vu de dos. Celui-ci indique bien sûr la Section XIX ${ }^{\circ}$ siècle du Musée d'art moderne. D’un musée historique récent consacré aux objets ayant appartenu aux souverains belges (Musée de la Dynastie), on passe donc à la fiction de musée forgée par l'artiste. Broodthaers, toujours équipé de son postiche, charge ensuite une caisse du Musée dans un camion de la Continentale Menkes, une compagnie spécialisée dans le transport d'œuvres d'art, puis il pose pour une photographie-souvenir. Viennent ensuite quelques vues prises depuis le camion sur des monuments historiques de la capitale belge (le Palais royal, la statue de Godefroy de Bouillon, le Palais de justice) et, enfin, Waterloo à nouveau. Si on n'échappe pas à quelques plans sur la morne plaine, Broodthaers fait surtout apparâttre le site grâce aux panneaux de signalisation routière qui désignent les lieux de la bataille (« QG Wellington/Mémorial britannique », « Ambulance britannique »), aux bâtiments historiques (la ferme de la Haie Sainte) et aux différents monuments qui célèbrent vainqueurs (la Butte du Lion, élevé par les Pays-Bas en 1826) ou vaincus (le monument à l'Aigle blessé, édifié par les Français). Surtout, devant la ferme de MontSaint-Jean (l'«Ambulance britannique ») apparaît un porte-drapeau, seule et unique figure de «reconstituteur» que l'on croisera dans toute l'œuvre de Broodthaers. Ce marcheur, qui rejoue très certainement quelque chose de l'épopée napoléonienne, nous l'avions en fait déjà aperçu en ville, devant le palais de justice. Le drapeau est en réalité une culotte placée sur une hampe ${ }^{19}$, et l'homme porte des habits contemporains. Ce personnage sur lequel le film ou l'artiste ne nous donne absolument aucune information, traverse la chaussée et s'engouffre dans la ferme située de l'autre côté. Enfin, une voiture de sport arrive dans un stationnement de Waterloo. En descend Marie-Puck, la jeune fille de l'artiste à qui le film est dédié.

Le film constitue la seconde partie du film Musée d'art moderne, Département des Aigles, Section $1 g^{e}$ siècle, 1969.

19 Voir Theys, 20oI, non paginé. 
Avec le premier acte du Musée des Aigles, le Voyage constitue le point de départ de la réflexion plastique de Broodthaers sur la reconstitution du passé historique. Le voyage tel que représenté dans le film est en fait une extension du Musée : d'abord, parce que le film met en scène un camion de la Continentale, dont le jumeau était stationné devant l'une des fenêtres de la rue de la Pépinière lors de l'inauguration du Musée; d'autre part, parce que Waterloo, c'est Napoléon et que l'imagerie napoléonienne, grande pourvoyeuse d'aigles en tout genre, est dès le départ un des appuis essentiels du Musée des Aigles. Le Voyage est aussi pour Broodthaers l'occasion de faire jouer sa fiction d'institution comme un révélateur, en dehors de ses propres murs. Désormais - et c'est ce dont l'artiste prend acte - l'Histoire du $19^{\mathrm{e}}$ siècle connaît de nouvelles formes de réification qui passent par la reconstitution, et il suffit d'aller quelques kilomètres au sud de Bruxelles pour observer ce spectacle.

\section{WATERLOO OU LA BATAILLE DU COMMERCE, DES ORIGINES À NOS JOURS}

« La célébration du bicentenaire de Napoléon a provoqué le déferlement d'un lot d'écrits à la louange de Napoléon, qui semble véritablement avoir effectué un nouveau retour et débarqué triomphalement sur les rivages de l'actualité2o ${ }^{2}$ »

Waterloo, dernière bataille de Napoléon et épisode final des Cent-Jours, est un mythe du $19^{\mathrm{e}}$ siècle, mais du $20^{\mathrm{e}}$ siècle tout autant, et ce, notamment grâce à la muséification du site et à son exploitation commerciale qui commence dès que le canon a fini de tonner. L'après-Waterloo voit se développer les prémisses d'un tourisme de la mémoire. Comme le rappelle A. V. Seaton, les touristes affluent sur le site immédiatement après la bataille à la recherche d'objets-souvenirs, des monuments ne tardent pas à être érigés par les divers États impliqués dans le conflit avec feu l'Empereur, et Waterloo est prestement intégré dans les itinéraires des premiers tour operators, celui du Britannique Thomas Cook par exemple. Les équipements culturels et commerciaux vont se multiplier. En I835, un ancien soldat anglais de Waterloo, Edward Cotton, ouvre le premier hôtel du site, doublé d'un petit musée de reliques, Le Grand Hôtel du Musée de Waterloo. Des visites guidées sont rapidement mises en place par des acteurs mêmes de la bataille (Cotton par exemple), alors que d'autres sont proposées par des habitants de la région qui s'arrogent le statut de

20 Maurice Guerrini, «Napoléon humain », Souvenir napoléonien, $\mathrm{n}^{\circ} 243$, spécial bicentenaire $\mathrm{n}^{\circ} 2$, septembre-octobre 1969, p. Iо. 
témoins de Waterloo, sans pourtant avoir vu quoi que ce soit ${ }^{2 \mathrm{I}}$. C'est encore une peinture panoramique réalisée par Louis Dumoulin qui est inaugurée en 1912, dans une rotonde située au pied du monument au Lion. À ces appropriations culturelles (les monuments) et commerciales s'ajoute la construction d'un mythe par la publication d'une avalanche de mémoires, d'histoires militaires, mais aussi d'une littérature poétique et romanesque mettant en scène un événement indescriptible en raison de sa complexité (Stendhal, La chartreuse de Parme, 1839) ou une bataille homérique (Hugo, Les misérables, 1862) 22. L'aura d'Hugo et celle de Waterloo se confondent bientôt, et le séjour que l'homme de lettres français fait sur le site en I86I à l'occasion de la rédaction d'une partie des Misérables donne lieu en I9ı2 à l'élévation d'un monument supplémentaire (terminé en 1956), la colonne Victor Hugo, et, ultérieurement (1962), à la muséification des restes de l'hôtel où il séjourna, l'Hôtel des Colonnes. Le champ de bataille devient lui-même patrimoine historique le 26 mars I9I4, par arrêté royal.

Waterloo est un objet parfaitement adapté pour le travail broodthaersien : c'est à la fois un objet du $19^{\mathrm{e}}$ siècle, un objet historique, un objet littéraire, un objet de musée et une chose commerciale à forte valeur ajoutée en l'année du bicentenaire de Napoléon. Il faut préciser ici que la décennie soixante fut une décennie napoléonienne : en 1968, c'est aussi le bicentenaire du rattachement de la Corse à la France et, en 1965, on célébra les I50 ans de la bataille de Waterloo. Ce dernier anniversaire, la première célébration à Waterloo depuis la fin de la Seconde Guerre mondiale, est boycotté par une France gaullienne assez soucieuse du prestige national et plus concernée par la célébration de la Libération (1964); la Belgique lui emboîte le pas. C'est pourtant l'amorce d'une remise en marche de la « gadgeterie impériale ${ }^{23} »$. C'est aussi l'occasion de différentes reconstitutions historiques avec un certain écho médiatique, à une époque où le reenactment et la living history n'ont pas encore pignon sur rue à Waterloo. On en conserve différentes images, notamment grâce à la

${ }^{21}$ Voir A. V. Seaton, « War and Thanatourism: Waterloo I8I5-I9I4 », Annals of Tourism Research, vol. 26, n I, 1999, p. 130-158. Susan Pearce (« The Materiel of War: Waterloo and its Culture », dans John Bonehill et Geoff Quiley (dir.), Conflicting Visions: War and Visual Culture in Britain and France, c. 1700-1839, Aldershot, Ashgate, 2005) examine comment, à Londres, les reliques de Waterloo deviennent immédiatement des objets recherchés pour la création d'expositions spectaculaires.

${ }^{22}$ Voir l'anthologie de Loris Chavanette (dir.), Waterloo: acteurs, historiens, écrivains, Paris, Gallimard, 20I5.

${ }_{23}$ Voir, R., «La gadgeterie impériale. Ou Napoléon, c'est toujours payant », Le Crapouillot, hors-série « Napoléon, cet inconnu », 1969, p. 102-106. 
remarquable émission de l'ORTF, Présence $d u$ passé, qui va célébrer cet anniversaire à sa manière: en moquant ce commerce du passé et les premiers pas de la reconstitution napoléonienne pour ce qui est de la période d'après-guerre. Présence $d u$ passé va aussi constituer un moment clé pour la carrière médiatique $d u$ reconstituteur précoce qui nous intéresse ici, Norbert Brassinne.

Créée par Jean Chérasse, Jean Mauduit et Bernard Revon en 1965, Présence du passé a pour mission de succéder à La caméra explore le temps (1953-1964), une production phare dédiée à l'histoire, de la première grande époque de la télévision française ${ }^{24}$. Chérasse et ses collègues prennent à rebours les principes de cette émission à succès et vont contester l'idée de fiction propre à la reconstitution historique. Avec l'appui de réalisateurs souvent venus du cinéma, ils vont produire des émissions d'histoire fiables sur le plan factuel, mais où le présent de l'énonciation est sans cesse réaffirmé par toute une panoplie de dispositifs de mise à distance, mâtinés de beaucoup d'humour et d'ironie. L'émission est un échec public (elle sera supprimée en 1968), mais elle trouve quelques soutiens critiques notoires aux Cabiers du Cinéma ou dans Combat. Les trois premières livraisons, réalisées par Georges Dumoulin avec la participation d'Abel Gance, sont dédiées aux Cent-Jours ${ }^{25}$ et cherchent à apporter un regard contemporain sur l'événement alors que, depuis un siècle et demi, se sont accumulés les récits mythologiques et images d'Épinal (Saint-Napoléon), les fantasmes les plus divers et l'exploitation commerciale de la chose. Ces trois premiers actes, comme d'autres épisodes ultérieurs de la série, sont marqués de manière assez évidente par les procédés de déstructuration du récit et de la représentation initiés par certains réalisateurs de la Nouvelle Vague, et peut-être par Culloden (1964) du Britannique Peter Watkins. Les trois volets des Cent-Jours confrontent ainsi avec brio différents registres de documents : images anciennes, extraits d'un film de fiction, numéros de chant avec Jean Yanne, entretiens avec des historiens ou avec des quidams pénétrés d'histoire, visites de musées en compagnie de guides parfois très habités par les personnages qu'ils évoquent, quelques scènes proches de la fiction traditionnelle (souvent accompagnées d'une voix hors champ), et plusieurs exemples de « reconstitutions non dramatiques ${ }^{26}$ » sur lesquels nous allons revenir. Un point

${ }^{24}$ Sur La caméra explore le temps et Présence du passé, voir Isabelle Veyrat-Masson, Quand la télévision explore le temps. L'bistoire au petit écran 1953-2000, Paris, Fayard, 2000, p. 89-113 et p. II9-I25.

${ }^{25}$ Les Cent-Jours, $\mathrm{n}$ et b, son, diffusé sur la $\mathrm{I}^{\text {re }}$ chaîne française. L'île d'Elbe, 74 minutes, Io octobre 1965; Le vol de l'Aigle, 73 minutes, 19 octobre 1965; 80 jours de sursis, 80 minutes, 25 octobre 1965.

${ }^{26}$ Voir Pierre Cabanne, « Adieu à Présence du passé », Combat, mardi 24 décembre 1968. 
marquant de cette livraison de la série est qu'elle met à jour la très grande diversité des acteurs de l'Histoire en cette période d'anniversaire historique. Les professionnels (historien de métier, conservateur d'un musée de province) y côtoient un membre d'une société d'érudition locale, un guide touristique ou encore la propriétaire d'une relique (une vieille dame qui possède un bol dans lequel Napoléon se serait rasé), de même qu'un certain restaurateur belge prompt à enfiler un uniforme napoléonien pour refaire les marches héroïques de l'armée napoléonienne. Il faut être franc, les différents représentants de cette histoire amateur ou vernaculaire ne sont pas à leur avantage. Loin d'être valorisé comme des acteurs de terrain développant des outils d'appropriation qui leur sont propres, ce petit peuple de l'Histoire est montré comme une horde assez grotesque, pétrie de croyances absurdes et préoccupée par son petit commerce. La charge contre la fiction historique populaire est aux antipodes de l'« Histoire par le bas » (History from Below) ${ }^{27}$ ! Et l'image de Waterloo, comme on va le voir, n'en sort pas indemne.

\section{LA RECONSTITUTION NAPOLÉONIENNE EN I 965}

« Lorsque je me ballade sur cette plaine, où papa a passé je dirais des milliers de fois, puisqu'il était déjà, lui, l'ermite du champ de bataille, eh bien, je dois dire que je revis $1815^{28}$. 》

Cohérents avec leur approche, Chérasse et ses collègues traitent la reconstitution historique comme une forme d'exploitation commerciale de l'Histoire et un outil publicitaire populaire ${ }^{29}$. Toutes les séquences qui y sont consacrées sont lestées d'ironie par la dramaturgie, la mise en scène et le montage. Même si Norbert Brassinne est le personnage central qui incarne la reconstitution historique dans l'émission et dont nous pensons qu'il joue un rôle dans l'intérêt de Broodthaers pour

${ }_{27}$ À partir de la fin des années 1950, différents historiens britanniques marqués à gauche (E.P. Thompson, Eric Hobsbawm) élaborent une « Histoire par le bas », en proposant une histoire sociale des $\mathrm{XVIII}^{\circ}$ et $\mathrm{XIX}^{\circ}$ siècles tournée vers les dominés. Les acteurs des luttes et organisations populaires y sont traités comme des agents rationnels et actifs de l'Histoire.

${ }^{28}$ Ces paroles sont prononcées par Brassinne dans le court métrage documentaire $W$ aterloo (Cousinet, 1985).

${ }_{29}$ Pour une vue contemporaine bienveillante sur les non-historiens qui entreprennent de raconter l'histoire, voir par exemple Jean-Noël Pelen, «Entre intime, histoire et Grands Récits : l'assemblement narratif du sujet », dans Maryline Crivello et Jean-Noël Pelen (dir.), Individu, récit, histoire, Aix-en-Provence, Publications de l'Université de Provence, 2008, p. II-34. 
Waterloo ${ }^{30}$, nous ferons néanmoins le choix de commencer par évoquer une autre séquence sans rapport avec le commerçant, mais qui, dans la chronologie de l'émission, intervient avant les épisodes dédiés à Brassinne. Cette première séquence nous donnera un aperçu d'une autre tentative de reconstitution napoléonienne, ainsi que de l'esprit caustique de Présence du passé.

La première séquence dédiée à la reconstitution apparaît à la fin du premier volet : Bonaparte, revenu d'entre les morts, débarque avec ses troupes sous le soleil de la Côte d'Azur, dans la France de 1965. Introduit par un carton signalant son caractère commémoratif, ce débarquement « refait », avec un public, a des allures d'actualités télévisées. L'événement, sans doute mis en scène par la municipalité de Golfe-Juan, ajoute lourdement le mythe au mythe : un texte nous informe en effet que Napoléon est « incarné » par l'acteur Albert Dieudonné, soit l'Empereur dans le film de 1927 Napoléon vu par Abel Gance. Ce rôle marquera tellement l'acteur qu'il s'identifiera à Napoléon jusqu'à la fin de sa vie. Gance, autre obsédé de Napoléon, est associé à la réalisation des Cent-Jours et propose tout au long du premier volet des réflexions sur la représentation qu'il se fait de Napoléon. Il est même interrogé par un journaliste sur la manière dont il filmerait Napoléon, assoupi après le débarquement sur les côtes françaises, comme si le réalisateur avait une telle proximité avec le grand homme qu'il pourrait faire des choix artistiques en connaissance de cause. Le réalisateur de Présence $d u$ passé ajoute une touche ironique finale au re-débarquement dans le port de GolfeJuan: le spectateur se voit en effet infligé l'écoute d'une interprétation de L'Ajaccienne (I848), un chant en l'honneur du retour de Bonaparte interprété par un autre Corse, Tino Rossi. Lorsqu'il entonne fiévreusement « La Sainte Famille est rentrée/ Les exilés sont de retour/ Les exilés sont de retour », le chanteur d'opérette fait basculer l'héroïque épisode de ce débarquement du côté d'un événement kitsch. Des scènes d'animation au dessin naïf finissent de liquider (discrètement) ce retour « refait » : des citoyens se mettent à genoux devant Napoléon, à son retour.

Norbert Brassinne (voir la figure I), lui, apparaît dans les deuxième et troisième volets de l'émission. Ce Wallon, dont la légende dit qu'il aurait changé son nom de Robert en Norbert pour arriver à concurrencer les initiales de Bonaparte, est un aubergiste de Braine-l'Alleud, une des communes qui constituent le site de la bataille de Waterloo. Issu d'une famille de tradition hôtelière liée depuis le $19^{\mathrm{e}}$ siècle au site de la bataille, Brassinne s'engage à son tour dans la carrière. Dès lors, il s'approprie la mémoire nationale française et développe des moyens de s’y intégrer.

$3^{\circ}$ Même si l'artiste n'a à peu près aucune chance d'avoir pu voir Présence du passé. En effet, d'après le dossier de production conservé à l'INA, ce programme n'a pas été vendu à l'étranger. 


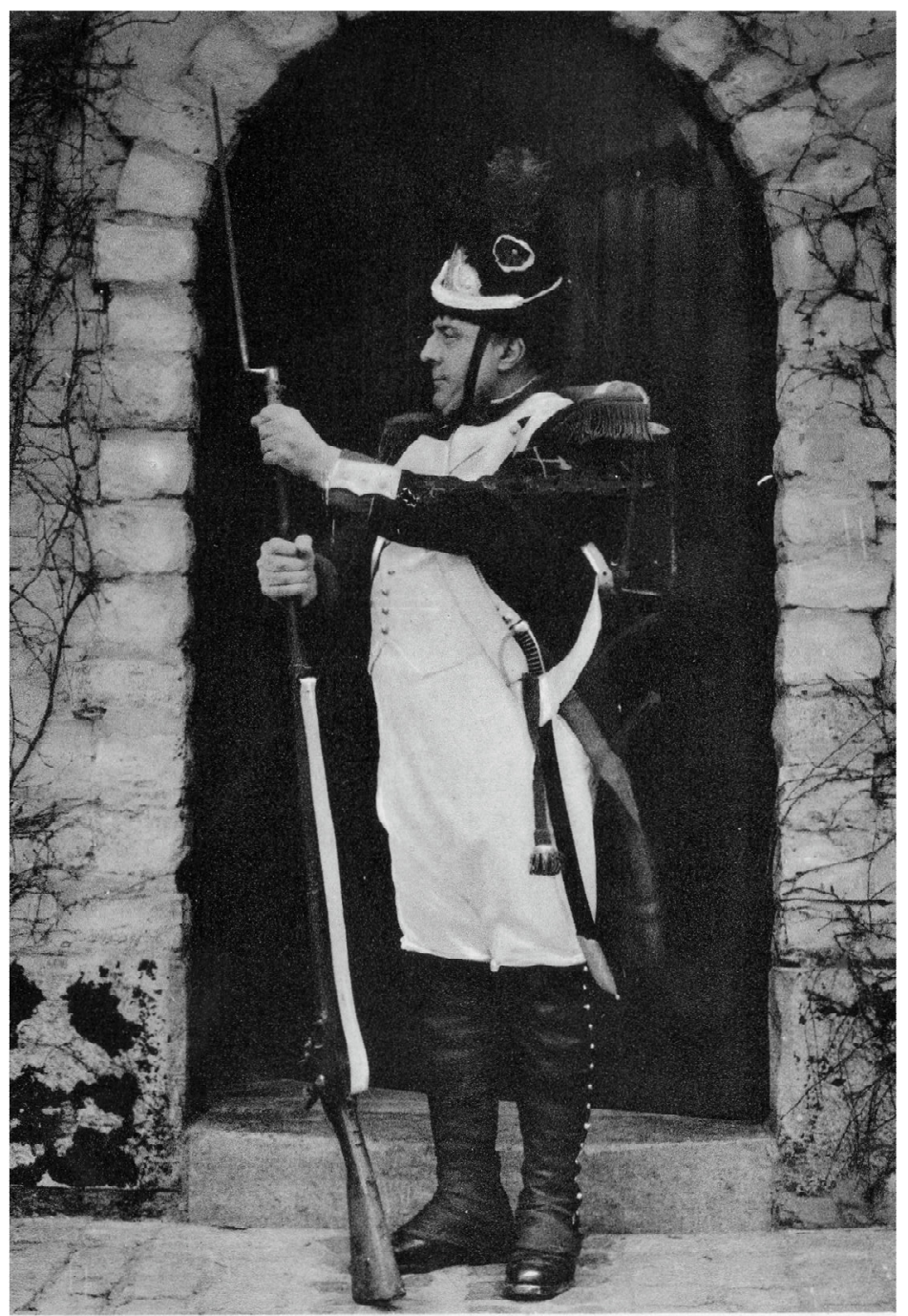

Le pélerin des Cent Jours, Norbert Brassinne, face à l'entréo de la Taverne - Restaurant du Bivouac de l'Empereur. Esplanade du Lion

Fig. I. Auteur inconnu, Carte postale commerciale produite par Norbert Brassinne/Restaurant Le Bivouac, 15 X I0, $5 \mathrm{~cm}$, collection personnelle, sans date (seconde moitié des années 1960).

Très tôt, il investit dans les outils populaires de représentation de l'Histoire. Il achète ainsi en 1947 une partie du Grand Hôtel du Musée de Waterloo dans laquelle il installe en 1949 quelques saynètes composées de figures de cire grandeur nature réalisées par des artistes du Musée Grévin. Ces tableaux proposaient au spectateur une vision du grand homme en pleine méditation, de ses généraux devant des cartes et des 
soldats de l'Empereur - une « histoire visuelle populaire et démocratique » visant entre autres «la dissolution de la distance au passé3I ${ }^{\mathrm{I}}$, dans la lignée du musée parisien. L'établissement de Brassinne avait aussi évidemment pour objectif de concurrencer la vision de la bataille offerte par le panorama de Louis Dumoulin et son faux-terrain, lui aussi excroissance directe des dispositifs de divertissement urbains du long $19^{\mathrm{e}}$ siècle. Au début des années 1950, Brassinne ouvre un restaurant au pied du Lion qui deviendra progressivement un symbole et un lieu de rendez-vous pour tous les reconstituteurs d'obédience napoléonienne, Le Bivouac de l'Empereur. Il possède aussi un cinéma où il montre des extraits de la version sonore du film de Gance. En 1965, à l'occasion du cent cinquantenaire des Cent-Jours, l'homme alors âgé de 57 ans, vêtu comme un grenadier à pied de l'Empereur, va proposer pour la première fois de sa carrière une curieuse « reconstitution » solitaire, non pas de la bataille de Waterloo, mais du parcours que réalisa Bonaparte avec son armée en I8I5, de Golfe-Juan à Paris, le long donc de ce que l'on appelle depuis 1932 la route Napoléon. C’est une reconstitution en un sens évidemment très large, puisque « le dernier grognard » (un de ses surnoms) ne cherche pas à mettre sur pied une armée, ni même à faire réapparaître l'Empereur, mais à mettre «ses pas dans les pas des grognards de l'Empereur », ainsi que l'énonce le commentaire hors champ de Présence $d u$ passé. Cette première marche est un petit événement médiatique en France, et les actualités françaises lui consacrent plusieurs sujets en 1965.

Présence du passé fait un portrait à charge de Brassinne, notamment dans sa seconde partie, en l'inscrivant dans une galerie de représentations de lieux où le commerce napoléonien bat son plein: l'île d'Elbe (traitée dans la partie I de l'émission), la route Napoléon (partie 2 de l'émission) et Waterloo, bien sûr (partie 3 de l'émission). Sur l'île d'Elbe, dont Napoléon fut l'éphémère souverain après sa disgrâce de I8I4, la présence de l'Empereur nous est rendue palpable surtout par la grâce des produits dérivés: figures, fioles, bouteilles d'une improbable cuvée impériale... La route Napoléon est, quant à elle, un enjeu commercial de taille pour petits et grands entrepreneurs depuis le début des années 1930 : ces derniers ont parsemé le parcours de plaques commémoratives, restaurants... Brassinne, lorsqu'il emprunte la Route en 1965 , adopte face aux médias une posture qu'il tiendra jusqu'au

${ }^{31}$ Fondé en I882 à Paris, le Musée Grévin se veut une sorte de journal « vivant ». Ses scènes reconstituées renvoient autant aux vedettes de l'actualité du jour qu'à des personnages plus historiques (Napoléon, bien sûr, ou Charlotte Corday). Pour les citations originales, voir Vanessa Schwartz, Spectacular Realities. Early Mass Culture in Fin-de-Siècle Paris, Berkeley, Los Angeles, Londres, University of California Press, 1998, p. 142. 
bout: il récuse toutes les implications commerciales de son geste, il se défend de vouloir se faire de la publicité par son action. Comme il le confie à un journaliste, il est «le représentant de quelque chose de plus grand». Devant les caméras des actualités télévisées françaises ou à la radio, le savoir devient généralement un outil d'autodéfense pour fonder la légitimité de ses «reconstitutions»: il évoque sa bibliothèque ${ }^{32}$, il cite beaucoup et avec force théâtralité (Victor Hugo, par exemple) et il dédie même sa marche à un héros de la littérature théâtrale, Jean-Pierre Séraphin, dit Le Lampard, le grognard fidèle de la célébrissime pièce d'Edmond Rostand, L'Aiglon (1900) 33. Brassinne veut s'inscrire dans l'espace du savoir et du mythe, historique et littéraire tout à la fois, de même que de la gratuité : il répète à qui veut l'entendre qu'il perd de l'argent dans cette affaire. Il joue donc l'ethos du passionné désintéressé, pour reléguer à l'arrière-plan son rapport au commerce. D’autres acteurs de la trilogie de Présence du passé sont plus francs sur les enjeux mercantiles de leur fièvre napoléonienne. Monsieur Bondil, secrétaire du Syndicat d'initiative de la route Napoléon, affirme ouvertement : «La route Napoléon doit être commercialisée. » Au journaliste qui lui demande : «Et vous, est-ce que vous êtes un fervent admirateur de la figure de Napoléon ? », l'homme répond : «En tant que commerçant, oui. » Dans ce contexte, sans mot user, la reconstitution de Brassinne est présentée comme une véritable petite allégorie du commerce, et l'homme lui-même, comme une sorte d'homme-sandwich de l'Empereur ${ }^{34}$. Quant au Waterloo contemporain, Présence $d u$ passé, comme les actualités télévisées belges et françaises de l'époque, le représente sous les traits d'une fête foraine. Dans le troisième volet de la trilogie, la séquence Waterloo 1965 nous fait apercevoir successivement le bâtiment du panorama de I9ı2, un cinéma, le fameux restaurant Le Bivouac et son aire de jeux pour enfants - le tout accompagné d'un extrait crachotant de la visite guidée du Naporama créé par Brassinne, «le nouveau et le plus beau musée didactique du monde 35 ». Après

32 《Robert Sadoul interviewe un "grognard belge" », sujet diffusé dans Inter actualités, I3 h oo sur France Inter, radiodiffusé le 9 mars 1965.

33 «Le Grognard », sujet diffusé dans le Journal de Paris sur la $\mathrm{I}^{\text {re }}$ chaîne française le 20 mars 1965 .

${ }_{34}$ C'est le cas aussi de la très sérieuse Société belge des études napoléoniennes. Voir le bulletin de la Sben, $\mathrm{n}^{\circ} 52, \quad 1965, \quad$ p. I4-I5. Disponible en ligne: www.sben.be/pdf/N52_1965_Commemoration_I50_ans_p_5_22.pdf (consultation le 26 mai 20I7).

35 Une affiche non datée (accessible en ligne : http://waterloolionrenovation.weebly.com) propose cette description sans appel. Les Actualités françaises, édition pour l'étranger (Belgique) du journal, consacrent le I4 septembre 1960 un sujet à ce musée («Un Naporama 
quelques doctes explications et la reconstitution de la bataille avec des soldats de plomb par un club anglais de Southampton, les appareils de divertissement sont à nouveau les héros d'une séquence intitulée « Ce que devient l'histoire passant par le commerce... ». Et voici encore la longue cohorte des restaurants, des cinémas, des reliques publicitaires utilisées par Brassinne pour décorer les alentours de son établissement (une calèche) et encore une réclame pour une bière Napoléon, spécialement réalisée pour l'anniversaire de la bataille. On aperçoit aussi une statue de Bonaparte, sur un socle, tenant une longue-vue, et que Brassinne avait baptisée « la borne centrale de l'Europe » : toute distance à un point situé sur le globe terrestre devait, selon le restaurateur, être calculée à partir de la statue ${ }^{36}$. Vient enfin Brassinne lui-même, en civil et paré du titre de président du Syndicat d'initiative du Lion de Waterloo. Pour les journalistes, il réalise une visite guidée hautement pathétique de la ferme d'Hougoumont. La série se clôture sur cette inénarrable parole d'un homme qui, tel un Michelet de Waterloo, essaie de ressusciter les souvenirs enfouis dans les pierres et le verger de la ferme.

\section{NORBERT BRASSINNE OU LES AGENDAS DE LA RECONSTITUTION HISTORIQUE}

« Le vrai tourisme, c'est garder l'empreinte du passé. Et, comme disait Jean Jaurès, en écartant seulement les cendres ${ }^{37}$. »

Brassinne est une figure dont les actions liées à la reconstitution procèdent d'agendas multiples. Le discours qu'il élabore à propos de ses pratiques de reconstituteur et de commerçant tend pourtant à simplifier la donne et à construire le mythe d'un homme tout entier dédié à l'Histoire. Brassinne, dans cette perspective, peut se prévaloir d'un engagement politique au présent. Il est en effet un fervent militant wallon, pacifiste, inscrit dans différentes organisations politiques qui défendent avec passion leur territoire et leur francophonie, appelant à une organisation fédérale de la Belgique. Amoureux d'une certaine idée de la France, Brassinne puise la plupart de ses grandes thèses historiques et politiques dans le

Waterloo »), composé d'une succession, façon panorama, de peintures naïves sur la vie de l'Empereur.

${ }^{36}$ On apprend ce détail dans un sujet précoce diffusé par les Actualités françaises ( « Anniversaire Waterloo : le fana de Napoléon ») le I9 juin 1963, sur la $\mathrm{I}^{\text {re }}$ chaîne française.

37 Ces paroles sont prononcées par Brassinne dans le court métrage documentaire Waterloo (Cousinet, 1985). 
réservoir de thèmes des sociétés wallonnes pro-françaises d'après-guerre. Napoléon et Waterloo jouent un rôle important dans la rhétorique de ces organisations, comme nous allons le voir. Les Wallons pro-France ont ainsi associé la figure du général de Gaulle à celle de Napoléon et de Waterloo dès l'appel du I8 juin $1940^{38}$. C'est le cas de Brassinne, qui manifeste aussi une grande ferveur pour le général, érigé au rang d'exemplum contemporain. Jean-Émile Humblet, qui prononça l'oraison funèbre de l'aubergiste, nous apprend que Brassinne créa dans les années 1970 « la tradition du pèlerinage de Colombey-les-Deux-Églises, remorquant chaque mois de novembre $\mathrm{du}$ Brabant-Wallon à la "ligne bleue des Vosges une énorme croix de Lorraine avant de monter la garde toute la nuit devant le tombeau du général de Gaulle le jour anniversaire de son décès 39 ». À la geste napoléonienne répond, tel un écho, le grand récit élaboré en France dans l'après-guerre autour de la figure du général et de la France résistante. Et on ne pourra s'empêcher de penser que les hommages récurrents de Brassinne à l'Empereur, au Dôme des Invalides, sont comme des surgeons du rituel d'invention de la mémoire héroïque de la France gaullienne : le transfert des cendres de Jean Moulin au Panthéon en $1964^{40}$. Brassinne intègre aussi l'épopée napoléonienne dans ses actions pour la défense des intérêts culturels et économiques wallons. Ainsi, dans les années I970, pour intervenir dans les conflits qui opposent communautés francophones et communautés flamandes ${ }^{41}$, Brassinne paie de sa personne en organisant des actions politiques dans lesquelles il lui arrive parfois de paraître en costume de grenadier ou d'insurgé de i830 (date de l'indépendance de la Belgique $)^{42}$. Napoléon ne sert pas simplement d'étendard à une revendication identitaire très localisée: en effet, on le retrouve aussi dans l'argumentaire de politique internationale développé par Brassinne, très marqué, encore une fois, par

${ }^{38}$ Sophie Jaminon, «Waterloo », dans Paul Delforge, Philippe Destatte et Micheline Libon (dir.), Encyclopédie du Mouvement wallon, Namur, Institut Jules Destrée, 20oI, t. III, p. 1665 .

39 Jean-Émile Humblet, « Norbert Brassine (1907-1988)», Revue du sowvenir napoléonien, $\mathrm{n}^{\circ} 363$, février 1989 , p. 14.

$4^{\circ}$ Sur le transfert et son rôle dans la construction mémorielle de la France gaullienne, voir Henry Rousso, Le syndrome de Vichy, de 1944 à nos jours [1987], Paris, Éditions du Seuil, coll. « Points », I990, p. I00-II7.

${ }^{41}$ Dans les années 1960, Brassinne n'aborde pas le sujet face aux médias français. Quelques discussions sur ce point ont lieu dans le beau documentaire de Nathalie Perette, Le chant $d u$ grognard, tourné en 1980 et diffusé le 13 janvier 1982 sur la $\mathrm{I}^{\text {re }}$ chaîne française.

${ }^{42}$ Voir Paul Delforge, « Norbert Brassinne », dans Paul Delforge, Philippe Destatte et Micheline Libon (dir.), Encyclopédie du Mouvement wallon, Namur, Institut Jules Destrée, 2000, t. I, p. $195-196$. 
les grandes orientations des Wallons pro-français des années 1950 et 1960. Loin d'appeler à unifier la France et la Wallonie comme c'était le cas avant la Seconde Guerre mondiale, les Wallons pro-français d'après-guerre veulent concevoir une Wallonie indépendante, « légitimée par les autorités internationales » et reconnue par une «Europe fédérée ${ }^{33}$ ». Brassinne, devant les caméras de télévision, se déclare plus d'une fois être le valeureux héraut des États de l'Europe unie, dont la défaite de Waterloo aurait, selon lui, empêché l'avènement. C'est un Napoléon revisité (pour ne pas dire « réinventé ») qu'utilise Brassinne pour fonder son discours pro-européen : loin du conquérant, c'est bien plutôt un homme dédié à l'union et à la paix que dépeint le restaurateur et reconstituteur de Braine-l'Alleud. À son échelle, il essaie d'ailleurs de reprendre en main ce projet européen en créant un début de réseau transnational dédié à la mémoire de l'Empereur: en 1966, il voyage en Tchécoslovaquie communiste jusqu'au champ de bataille d'Austerlitz et inaugure ainsi un pèlerinage qui connaîtra plusieurs occurrences. Difficile malgré tout de ne pas sourire en voyant notre homme s'extraire de sa voiture, à Paris, peu de temps après la crise étudiante de 1968 et en pleine époque flower power, avec son costume et la violette au fusil (signe de paix) ${ }^{44}$.

L'appropriation de la geste nationale française par le citoyen belge ne se cantonne pas, on l'a déjà noté, à une appropriation à caractère politique. Comme le sumbolon a deux faces, Brassinne est aussi le roi du produit dérivé et de l'attraction à coloration historique. Sa réactivation du personnage du vieux grognard - une représentation solidement construite par les images d'Épinal et la réclame tout au long $\mathrm{du} 19^{\mathrm{e}}$ siècle - est efficacement médiée par une ribambelle d'événements publicitaires, de cartes postales commerciales, d'affiches et de reportages télévisés. Son grognard renvoie à une vision de l'histoire des plus scolaires, bien sûr totalement adaptée à l'industrie du tourisme historique : l'Histoire est d'abord et avant tout le storytelling du grand homme, des grands événements et des preux soldats dédiés à la cause - le reconstitueur en est d'ailleurs leur alter ego, gardien du flambeau tout entier engagé. Dans le cadre de son entreprise mémorielle-commerciale, Brassinne fait aussi main basse sur le vocabulaire de l'invention de la tradition : passé personnifié (il se désigne comme le « pèlerin de la paix » ou « des Cent-Jours »), fétichisation des

43 Jaminon, 200I, p. 1665. Pour mémoire, la Communauté économique européenne (qui incluait la Belgique) est fondée en 1957.

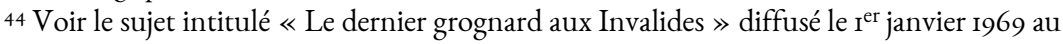
Journal télévisé de la nuit, $\mathrm{I}^{\mathrm{re}}$ chaîne française. 
lieux et des dates de l'histoire (voir ses cartes postales estampillées d'un cachet anniversaire), attribution d'un rôle fondamental aux commémorations et aux monuments ${ }^{45}$, ou encore instauration de reliques. Ainsi, sur une des innombrables cartes postales commerciales produites par l'aubergiste ${ }^{46}$ (ici, en 1965 ) on apprend que l'homme a fait ramener de la «terre sacrée » de Waterloo à Golfe-Juan. Le refaire brassinnien puise en fait indifféremment à des modèles extrêmement différents d'écriture de l'histoire : historia magistra (le grand homme inspirant les plus hautes vertus que le lecteur devrait chercher à imiter), histoire positiviste (revendication de l'exactitude et de l'objectivité dans la reconstitution ou le costume), invention de la tradition. L'ensemble constitue la fondation d'une culture autochtone de la mémoire, mais aussi d'un redoutable appareil de promotion où la reconstitution joue un rôle clé. La reconstitution peut d'ailleurs intégrer le client, comme sur cette autre carte postale publicitaire (1965), où Brassinne invite qui le voudra à venir « manger et boire comme en I8I5 » pour célébrer son retour à Waterloo, à la suite de son excursion en France sur les traces de Napoléon. Une autre carte postale, de 1968 celle-ci, associe, elle, plus littéralement «reconstitution» et réclame: Brassinne, sur une photographie datée du I8 mai (sans doute faut-il lire juin) I968, apparaît « en tenue de demi-solde devant le Lion de l'Empereur », non loin d'un petit encart publicitaire vantant une marque célèbre de « baume bains de pieds poudre ». In fine, le refaire tel qu'envisagé par Brassinne peut assumer des fonctions différentes (commémorative, politique, promotionnelle) selon les contextes, mais pas exclusives les unes des autres. Si le succès commercial est au rendez-vous, et si Brassinne finira par transmettre la passion de la reconstitution à quelques héritiers ${ }^{47}$, les grands médias considèrent cet empire commercial avec distance et ironie, que l'on regarde du côté des grands hebdomadaires papier du milieu des années $1960^{48}$ ou du côté de la télévision française (qui fait du reconstituteur pittoresque un sujet récurrent entre

45 Un sujet déjà cité des Actualités françaises («Anniversaire Waterloo: le fana de Napoléon ») nous apprend que Brassinne va chaque i 8 juin déposer une gerbe de coquelicots et de bleuets cueillis à Waterloo sous l'Arc de Triomphe à Paris.

${ }^{46}$ Les cartes postales évoquées dans ce paragraphe ont été glanées sur le site de vente en ligne Delcampe : www.delcampe.net/en_US/collectibles/ (consultation le 27 mai 2017).

47 Voir le film $W$ aterloo (Colinet, 1985).

${ }^{48}$ Voir Roger Mauge, « Napoléon gagne à Waterloo », Paris Match, $\mathrm{n}^{\circ} 846,26$ juin 1965 , non paginé; Peter von Brügge, « Nach den Preussen diesmal nicht gerufen », Der Spiegel, $\mathrm{n}^{\circ} 26,2 \mathrm{I}$ juin 1965 , p. 28-29. 
I965 et 196949). En 1965 notamment, les journalistes de Paris Match ou de Der Spiegel semblent nettement préférer l'austérité des célébrations britanniques, plus en accord avec les normes de la culture officielle de la mémoire. Une seule image échappe à cet ensemble : peu avant la célébration des I5o ans de Waterloo, notre grognard apparaît en marcheur héroïque sur la couverture d'un supplément à Paris Match destiné à la zone Benelux (voir la figure 2). Mais elle parait être restée l'unique en son genre.

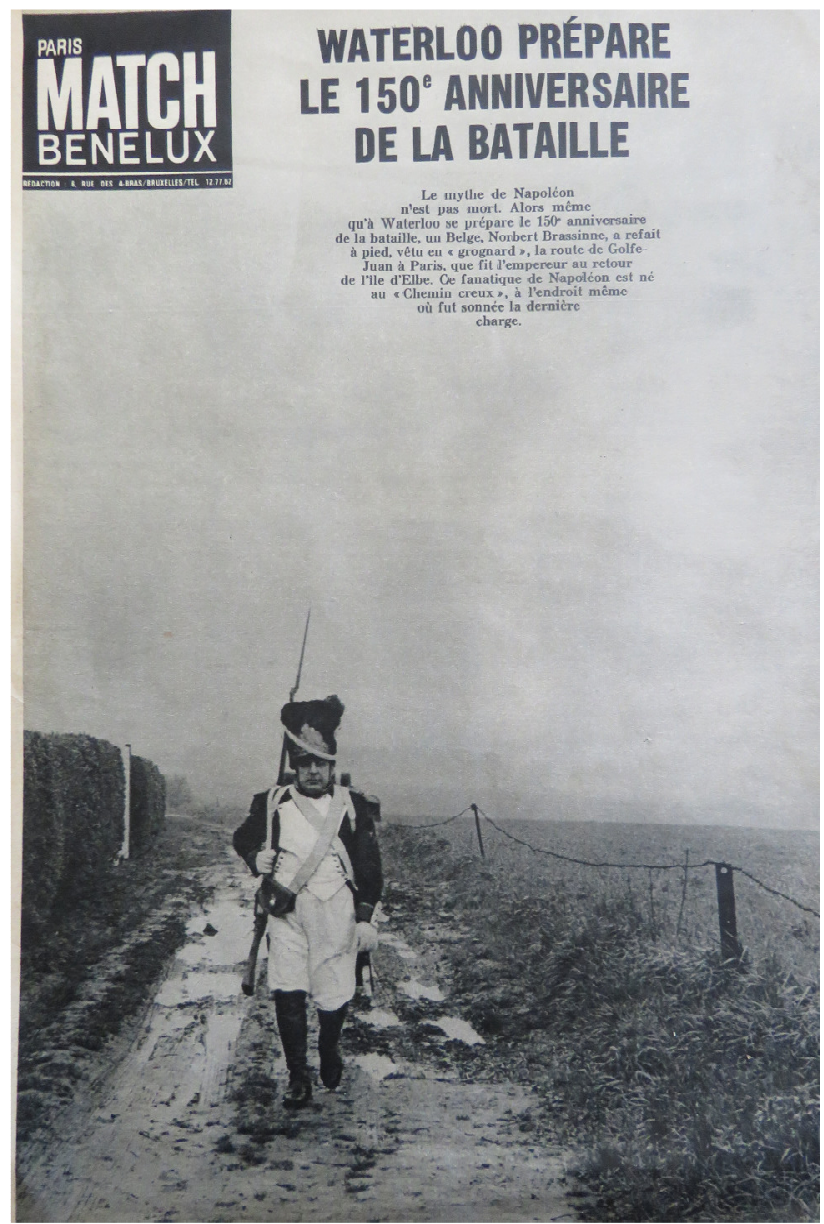

Fig. 2. Couverture du supplément Bénélux de Paris Match, $35 \times 27 \mathrm{~cm}$, collection Véronique Denis, juin 1965. (C) Christian Gibey, 1965.

49 La télévision belge ou les actualités Belgavox ne semblent, elles, presque pas s'intéresser au phénomène. Par manque de place, nous ne proposons ici qu'une synthèse rapide de nos recherches dans les fonds de l'INA, de la SONUMA et de Belgavox. 


\section{TU N'AS RIEN VU À WATERLOO}

« [...] et tout cela pour qu'aujourd'hui un paysan dise à un voyageur : Monsieur, donnez-moi trois francs; si vous aimez, je vous expliquerai la chose de Waterloo ${ }^{50}$. »

Le Waterloo de Brassinne et celui de Broodthaers sont fondamentalement divergents. Nul signe d'intérêt chez l'artiste pour le tissu de revendications régionalistes wallonnes, nulle fascination pour l'idée d'une « contre-histoire ${ }^{5 I}$ » vernaculaire ou d'une histoire locale que se seraient appropriés ses acteurs mêmes. L'épistémologie et l'époque n'en sont pas encore à s'interroger sur les usages publics de l'histoire, et, qui plus est, Broodthaers, en bon héritier d'un champ littéraire $19^{\mathrm{e}}$ siècle ordonné autour de l'opposition industriel/autonome, a une certaine tendance à disqualifier toute pratique ou forme ne lorgnant pas du côté de l'autonomie. Quoi qu'il en soit, avec sa carte postale de 1969 (un film réalisé « à titre de souvenir personnel », dit-il), Broodthaers inaugure une enquête au long court sur les croisements entre folklore, nationalisme, commerce et tourisme en Europe. Le Musée des Aigles sera un lieu essentiel pour l'avancée de ladite enquête, notamment la Section des figures et la Section publicité. Bien que proposant un cadre historique extrêmement large, ces deux sections sont tout de même largement focalisées sur le moment romantique européen et la culture de masse d'après-guerre. La Section des figures (Düsseldorf, 1972) met en scène la circulation du symbole de l'Aigle à travers des objets d'époques très diverses et fait la part belle à son devenir symbole nationaliste dans les imaginaires politiques de l'Europe moderne et contemporaine; la Section publicité (Kassel, I972) vient jouer le rôle d'archive de la précédente ${ }^{22}$ et transforme les images de Düsseldorf en documents, réifiant encore un peu plus ce symbole de pouvoir. Cette section est aussi l'occasion pour Broodthaers de pointer l'intégration massive de la figure de l'Aigle dans la communication publicitaire

so Victor Hugo, Les misérables [I862], édition présentée, établie et annotée par Yves Gohin, Paris, Éditions Gallimard, coll. « Folio classique », 1999, t. II, p. 438.

51 Voir Maryline Crivello et Nicolas Offenstadt, «Concurrences et controverses. Introduction », dans Maryline Crivello, Patrick Garcia et Nicolas Offenstadt (dir.), Concurrence des passés. Usages politiques du passé dans la France contemporaine, Aix-enProvence, Publications de l'Université de Provence, coll. «Le temps de l'histoire », 2006, p. 194.

52 Sur le lien de la Section publicité avec le romantisme, voir Marcel Broodthaers, « Museum für moderne Kusnt. Abteilung die Adler », Heute Kunst, Milan, nº I, avril 1973, traduit dans Catherine David et Véronique Dabin (dir.), Marcel Broodthaers, Paris, Éditions du Jeu de Paume, 1992, p. 227. 
contemporaine (voir par exemple les nombreux documents relatifs à la ville suisse d'Aigle). La section du musée qui devait être dédiée au folklore, la Section folklorique/Cabinet de curiosités (Zeeuws Museum, Middelburg, 1970), n’aboutira finalement pas. C'est en fait surtout dans les derniers travaux de Broodthaers qu'il faudra aller chercher les suites de l'enquête initiée à Waterloo en 1969 : le magnifique livre d'artiste En lisant la Lorelei (1975), qui met en tension les récits inventés du Rhin romantique 53 , ou, bien sûr, l'installation Décor : A Conquest (1975), sur lesquels nous reviendrons plus bas. Pour l'instant, reste à savoir si Broodthaers le Bruxellois connaissait les actions de Norbert Brassinne le Wallon. Sur ce point précis, la question reste ouverte. Le site marchand de Waterloo était, quant à lui, connu de Marcel Broodthaers ${ }^{4}$. Ainsi, dans l'une des versions du Voyagess, un plan fixe la façade du « cinéma de la bataille » appartenant à l'Hôtel des Alliés, un des établissements phares de la commune de Braine-l'Alleud. Une photographie prise pendant le tournage par Maria Gilissen montre, quant à elle, l'artiste posant fièrement devant un aigle napoléonien que Brassinne avait installé devant son restaurant ${ }^{56}$. On ne pourra donc s'empêcher de penser à Brassinne en voyant l'homme au drapeauculotte : dans les années I960, l'aubergiste a construit sa célébrité sur ses marches entre Golfe-Juan et Paris (parfois accompagné de son auto57), et sur ses pèlerinages, à Austerlitz (alors en Tchécoslovaquie) à partir de 1966 ou à Borodino (Russie) en pleine guerre froide. Mais le porte-drapeau de fortune - certainement un ersatz de porte-aigle napoléonien - pourrait tout aussi bien être un proche cousin de Brassinne : l'un de ces fameux marcheurs d'Entre-Sambre-et-Meuse qui, à certaines périodes de l'année, accompagnent des défilés religieux, revêtus des uniformes du Premier ou du Second Empire ou encore de l'époque de Léopold I ${ }^{\text {er }}$ ou Léopold II. Ces compagnies de marcheurs connaissent un véritable renouveau dans les années 1960 - certaines s'inventant ex nibilo - et elles développent un discours sur

53 Voir essentiellement Marcel Broodthaers, Autour de la Lorelei, édition critique, établie et postfacée par Philippe Cuenat, Genève, Mamco éditions, 1997; et Jean-Philippe Antoine, Marcel Broodthaers, moule-muse-méduse : un essai critique en sept coups, Dijon, Les Presses du Réel, coll. « L'espace littéraire », 2006.

${ }^{54} \mathrm{Il}$ arrivait à l'artiste d'emmener ses invités visiter le champ de bataille. Voir le témoignage de Jürgen Harten dans Marcel Broodthaers. An Attempt to Retell the Story: projet pour un traité de toutes les figures en trois parties, Cologne, Verlag der Buchhandlung Walther Konig, 2015, p. 43-44.

s5 Borja-Villel, Compton et Gilissen (dir.), 1997, p. 84.

${ }^{56}$ Voir Borja-Villel, Cherix, et al., 2016, p. 172.

57 Anonyme, « Le Napoléon 1969 », Sisteron-Journal, samedi 8 mars 1969, non paginé. 
le renouveau et la protection du folklore (les marcheurs vont jusqu’à réfléchir à la protection légale des marches ou des uniformes). Leur bulletin trimestriel comporte des textes appelant à la défense de l'exactitude des costumes et des gestes, de l'authenticité folklorique et des identités locales ${ }^{8}$. Opposés à toute commercialisation, les marcheurs appellent dans le même temps à attirer autant que possible l'attention des médias (radio et télévision), s'engouffrant ainsi dans un espace plutôt difficile à maitriser. Le marcheur de Broodthaers pourrait, enfin, aussi évoquer l'un de ces fameux pèlerinages à Waterloo des Wallons pro-français. Inaugurés en 1928 (rapidement médiatisés par les actualités filmées et la radio), ils déclinent à la fin des années 1930 et sont réinstaurés en 195699 . Quel que soit le référent exact du personnage broodthaersien, ce dernier semble assez clairement s'inscrire dans une allégorie démontant l'idée de reconstitution, aux antipodes bien sûr du traitement de l'histoire et de la mémoire historique proposée par le restaurateur de Braine-l'Alleud ou, quelques années auparavant, par l'Expo 58 avec son village belge édifié pour l'occasion et ses démonstrations de traditions populaires. Loin de prétendre rejouer le passé au présent dans ces années 1960 de la société de consommation ${ }^{60}$, Broodthaers, dans certaines des versions du Voyage à Waterloo, transforme le porte-drapeau en fantôme en le faisant disparaître à la fin du film dans des nuages de fumée, non loin de vaches paissant paisiblement ${ }^{6 \mathrm{r}}$.

Avec Un voyage à Waterloo, Broodthaers poursuit sa recherche « d'une rhétorique se nourrissant au nouveau dictionnaire des idées reçues ${ }^{62} \gg$. Grâce à sa fiction de musée, l'artiste révèle le champ de bataille pour ce qu'il est : un musée des lieux communs, à ciel ouvert, dans lequel chaque lieu est indexé par un cartel, c'est-àdire des panneaux de signalisation. Broodthaers ressaisit tous les topoi du site dans sa fiction : il tamponne « Musée des Aigles ${ }^{63}$ sur des cartes postales du Monument au

$5^{8}$ L'Association des marches folkloriques d'Entre-Sambre-et-Meuse (fondée en 1960) est dotée d'un bulletin trimestriel, Le marcheur de l'Entre-Sambre-et-Meuse. Brassinne ne relève pas de la catégorie du marcheur au sens où l'entend l'Association, mais il retient néanmoins la curiosité du bulletin en 1965. Voir Anonyme, «La marche du souvenir », Le marcheur de l'Entre-Sambre et Meuse, n 17, 1965, p. 19.

59 Voir Jaminon, 200I, p. 1664-1665.

${ }^{60} \mathrm{Ce}$ qui ne l'empêche pas, dans la lettre à Jacques Charlier déjà citée (Theys, 200I, non paginé), d'exagérer volontairement l'importance de la dimension de reconstitution de l'opération : Broodthaers parle en effet d'un film « tourné avec des soldats ».

${ }^{6 r}$ Borja-Villel, Compton et Gilissen (dir.), 1997, p. 82.

${ }^{62}$ Voir « Marcel Broodthaers, d'après un entretien avec Irmeline Lebeer », dans Yves Gevaert (dir.), Marcel Broodthaers catalogue / Marcel Broodthaers Catalogus, Bruxelles, Palais des Beaux-Arts, 27 septembre-3 novembre 1974, p. 64.

${ }^{63}$ Sur ces cartes postales, voir Borja-Villel, Compton et Gilissen (dir.), 1997, p. 87. 
Lion et étiquette au nom de son institution le canon à côté duquel il s'assoit au début du film et même la hampe de notre porte-drapeau, ainsi que nous le révèlent des photographies prises par Maria Gilissen pendant le tournage du film. La figure du musée et du refaire élaborée par Broodthaers gagne en intelligibilité si on se tourne vers le cinéma d'art et d'essai français des années 1950-1960. Il faut se souvenir que le Belge commence sa carrière dans l'après-guerre en étant libraire, guide d'exposition, journaliste, poète bien sûr, mais aussi cinéaste. Le musée est une figure fondamentale du cinéma de court métrage documentaire français d'après-guerre. Dans les années 1950, Georges Franju, Chris Marker et Alain Resnais vont explorer chacun à sa façon le musée comme machine à fabriquer du récit, de l'idéologie et donc la mémoire du temps. Là où Franju dans Hôtel des Invalides (195I) déconstruit la grande geste des guerres mondiales en nous emmenant faire une visite guidée du Musée de l'armée à Paris, Resnais et Marker, avec Les statues meurent aussi (1953), démolissent subtilement l'enveloppe hermétique du Musée de l'Homme à Paris en confrontant les expôts de sculpture nègre aux conséquences de la colonisation sur les cultures africaines. À partir de 1955, la maison de production Argos films, fondée par Anatole Dauman, va rapidement devenir un haut lieu de thématisation de la figure du musée et, plus largement, des institutions de la mémoire et de la conservation. Si dans les productions Argos on connaît surtout les traitements courts et longs de Marker et Resnais, dont l'un au moins n'est pas insensible à l'appropriation des folklores par la société de consommation ${ }^{64}$, il y a aussi celui, moins connu, d'Ado Kyrou. Ce franctireur du surréalisme d'après-guerre, théoricien du cinéma et membre important de la revue Positif dans les années 1950, réalise en 1957 La déroute, un court-métrage sur Waterloo comme lieu de mémoire et de consommation ${ }^{65}$. Avec ce film, Kyrou, accompagné par Franju à la collaboration artistique, revisite l'Hôtel des Invalides du même Franju. Une voix hors champ composée à partir d'extraits de Victor Hugo déconstruit la mythologie héroïque de Waterloo et piétine l’héroïsme suggéré par la forêt de monuments qui ornent le champ de bataille. C'est surtout le petit village

${ }^{64}$ Muriel ou le temps d'un retour (Alain Resnais, 1963). Broodthaers évoque son admiration pour le film dans un brouillon de lettre (du 2 novembre 1963) à Jean Cayrol, auteur, éditeur, scénariste et cinéaste qui a écrit le scénario du film de Resnais. Voir la figure 7, Paris, Éditions Fourbis, 1992, p. 9.

65 Jean Chérasse, un des fondateurs de Présence du passé, reconnait l'importance des films de Kyrou, Resnais et Marker pour sa démarche. Voir Vingtras (pseudonyme de Chérasse), «L'exécrable commémoration », Blog Vingtras, I8 juin 2015, https://blogs.mediapart.fr/vingtras/blog/I8o6is/lexecrable-commemoration (consultation le 20 août 2016). Et, du même auteur, «Alain Resnais », Blog Vingtras, 3 mars 20I4, https://blogs.mediapart.fr/vingtras/blog/0303I4/alain-resnais (consultation le 20 août 2016). 
commercial situé au pied du Lion qui subit les assauts du duo Kyrou/Franju. Le spectateur découvre alors largement l'instrumentarium commercial de Brassinne (qui n'est jamais nommé ou montré). Surtout, on plonge dans les entrailles de son cinéma, puisque Kyrou incorpore une séquence du film de Gance dans son propre film et que nous accompagnons les spectateurs à leur sortie. «Touristes ... Ne quittez pas sans avoir vu en 20 minutes le film parlant de la bataille de Waterloo », nous dit une réclame ... Un peu plus loin, un plan capture les toits de ce qui est vraisemblablement l'hôtel des Alliés : y est peint « CINÉMA » en lettres géantes. Méta-commentaire sur les usages marchands du cinéma et visite guidée alternative du champ de bataille, $\mathrm{La}$ déroute fait entendre une musique bavaroise du plus mauvais goût, émanant d'un juke-box dans lequel jouent des automates musiciens ${ }^{66}$.

\section{BROODTHAERS OU LE REFUS DU REFAIRE}

«Mon propos est ici fragmentaire. Mesurer le temps en comparant des images avec leurs inévitables associations d'idées ${ }^{67}$. »

Si le cinéma du musée, dans toute sa diversité d'approches, infiltre bien l'approche broodthaersienne du champ de bataille, la critique du refaire historique élaborée par l'artiste est polymorphe et ne se cantonne pas au domaine du cinéma. De fait, après la réalisation du Voyage, la question de la reconstitution reviendra inlassablement dans le travail de Broodthaers, fondant à la fois son refus de toute identification définitive de l'œuvre et un modèle critique d'exposition - et du récit historique par là même. Waterloo accompagne l'artiste sur cette voie tout au long des années $1970^{68}$. Son Voyage à Waterloo, outre qu'il compte plusieurs versions, est remis en jeu par l'artiste lorsque celui-ci le réinscrit dans un des programmes de films dont il a le secret, celui de la Section cinéma du Musée en 1972. Le film dialogue alors avec des reportages sur la ville de Bruxelles, une bande d'actualités Belgavox consacrée à la mode, le logo animé de la $20^{\text {th }}$ Century Fox ou encore avec Charlot als Filmstar, dans lequel Chaplin incarne un prétendant au métier d'acteur, expulsé sans ménagement d'un studio de cinéma. Le corps de l'acteur britannique vient s'associer

\footnotetext{
${ }^{66}$ Musique signée par le compositeur belge surréaliste André Souris. Également tourné en 1957, le Waterloo du documentariste belge Edmond Bernhard (un autre cinéaste du Musée) s'attaque aussi au champ de bataille pour touristes.

${ }_{7}$ Broodthaers, 1997 , p. 83.

68 Année marquée par la production d'une superproduction cinématographique reconstituant la bataille de Waterloo : Waterloo, Bondartchouk, 1970.
} 
ici à celui du porte-drapeau de Broodthaers tout en proposant aussi une image de l'artiste en maniaque de la reconnaissance ${ }^{69}$. Plus conséquent encore, il refuse in extremis à la fin de 1975 de refaire à l'identique le Musée des Aigles version 1968 pour l'exposition parisienne L'Angélus de Daumier. Rejetant les modèles de la period room $^{70}$ et de la rétrospective, Broodthaers imagine alors la Salle Blanche, « un rez-dechaussée parfaitement petit-bourgeois où flottent les mots », notamment celui de « copie ». Il n'envisage alors la reconstitution qu'involontaire, c'est-à-dire signée par un autre que lui, donc sans doute posthume: «Je crois que je n'en ferai pas ou, en tout cas, je n'en signerai aucun $(s i c)^{71}$. $\gg$ Broodthaers allégorise encore l'impossible reconstitution dans l'une de ses dernières grandes œuvres : l'installation Décor: $A$ Conquest réalisée à l'ICA à Londres en 1975. Réalisée non loin de Waterloo Place et consacrée à Waterloo, cette installation fait s'affronter encore une fois $19^{\mathrm{e}}$ et $20^{\mathrm{e}}$ siècles. À chacun échoit en effet un espace, occupé par un ensemble de figures allégoriques. Si les canons français et anglais installés sur du faux gazon ont la part belle dans la Section $\mathrm{XIX}^{\circ}$ siècle, la Section $\mathrm{XX}^{\circ}$ siècle est, elle, occupée par des figures allégorisant respectivement le tourisme et le musée : quatre chaises et une table de jardin surmontée d'un parasol, d'une part, et, d'autre part, ce que l'on pourrait décrire comme un « musée d'armes » (une des nombreuses échoppes de Waterloo), à savoir des armes automatiques factices alignées les unes à côté des autres sur des étagères. Le film La bataille de Waterloo, tourné dans ce décor composé entre autres d'objets glanés dans un magasin d'accessoires pour le cinéma ${ }^{72}$, poursuit magistralement l'enquête amorcée par l'artiste en 1969 sur la commercialisation du folklore historique ${ }^{73}$. Sur un fond wagnérien (le très mythologique Tristan et Isolde) s'y croisent et s'y confrontent une bouteille de cognac ornée de la représentation de l'Empereur, la destruction d'un casse-tête figurant une peinture de Waterloo et des

69 Pour le programme de la Section cinéma, voir Borja-Villel, Compton et Gilissen (dir.), 1997, p. 145-153.

$7^{\circ}$ Voir Catleen Chaffee, « Chez Broodthaers : considérations domestiques », dans Marcel Broodthaers. La Collection des musées royaux des beaux-arts de Belgique, Gand-Courtrai, Éditions Snoeck, 2oIo, p. 84.

${ }^{71}$ Pour les deux citations, voir « C'est l'Angélus qui sonne. Entretien de Stéphane Rona avec Marcel Broodthaers », dans +-O, Bruxelles, Genval, 1976, republié dans Marcel Broodthaers par lui-même, édition établie et introduction par Anna Hakken, Gand, Amsterdam, Ludion, Flammarion, 1998, p. I32-I33.

${ }^{72}$ Voir Barry Barker, « Décor: A Conquest by Marcel Broodthaers. Préparatifs pour un film $\gg$, dans David et Dabin (dir.), 1992, p. 275 .

${ }^{73}$ Le film est produit par une société fictive : Le Lion Belge. 
plans sur le Trooping the Colour, défilé annuel dérivé d'un rituel de champ de bataille et qui marque l'anniversaire du souverain britannique. Cette théorie de mythes, ce rituel pittoresque et publicitaire ${ }^{74}$ et son environnement architectural (le Mall) édifié à une époque où la monarchie britannique réinventait ses fastes ${ }^{75}$ élargissent encore les murs du « décor » de Broodthaers, qui fait déjà office de musée des mythologies de la conquête et de la guerre dans la peinture d'histoire et le cinéma commercial. Par delà Décor lui-même, La bataille lorgne aussi du côté du Voyage : à la fin du film de I975 apparaît un homme assis dans l'escalier d'un véhicule ambulancier, en train de retrousser sa chemise, et l'on peut difficilement ignorer qu'un des lieux essentiels $\mathrm{du}$ champ de bataille de Waterloo était la fameuse « ambulance britannique » à MontSaint-Jean, filmée par l'artiste dans son opus de 1969. Revenons une dernière fois à $L a$ bataille. Broodthaers éclaire le Décor par une photographie publiée sur la couverture du bulletin de l'ICA correspondant à l'exposition : elle représente le Grand Hôtel du Lion, le panorama de Waterloo et le Monument au Lion ${ }^{76}$. Ces équipements commerciaux archaïques, typiques du $19^{\mathrm{e}}$ siècle industriel, ressurgissent une dernière fois dans l'œuvre de Broodthaers et viennent dessiner clairement la fonction du Décor : être comme une parodie d'un de ces outils à immortaliser et à vendre ce passé spectaculaire dont le $19^{\mathrm{e}}$ siècle, avec ses musées de cire, ses fantasmagories et autres panoramas, a été très friand77. Décor, magasin d'accessoires, Waterloo, musée en plein air et plaine du commerce : c'est toute une panoplie de machines à faire l'histoire qu'expose ou suggère Broodthaers, de celles que les historiens ne regardaient pas encore dans les années 1970, mais qui prendront une place fondamentale à partir des années 1980 dans l'espace de ce champ disciplinaire.

De Waterloo à Waterloo, du film de famille au film historique, de la fiction du musée à la critique d'une certaine écriture de l'histoire - histoire vernaculaire et tradition inventée tout à la fois : ce sont quelques-uns des chemins sinueux qu'aura suivis Broodthaers dans la période 1968-1976. En 1975, dans un implacable projet de

${ }^{74}$ Bruce Jenkins qualifie le Trooping the Colour de «political advertisting ». Voir Bruce Jenkins, «Un peu tard: citation in the Cinema of Marcel Broodthaers », dans Borja-Villel, Compton et Gilissen (dir.), 1997, p. 294.

75 Voir David Cannadine, «Le cas de la monarchie britannique », dans Hobsbawn et Ranger (dir.), 20I2, p. I5I.

${ }^{76}$ Voir Borja-Villel, Compton et Gilissen (dir.), 1997, p. 287.

77 Voir Maurice Samuels, The Spectacular Past. Popular History and the Novel in Nineteenth Century France, Ithaca, Londres, Cornell University Press, 2004. L'exploitation de la geste napoléonienne par les attractions spectaculaires et la littérature au $19^{\mathrm{e}}$ siècle est un des sujets centraux de l'ouvrage. 
préface destinée à son En lisant la Lorelei, l'artiste formule nettement les enjeux qui nous auront intéressé ici. S’appropriant les récents propos de Michel Foucault sur la fascination nouvelle pour l'Occupation à la télévision et dans le cinéma français ${ }^{78}$, il signale l'affection de l'Europe contemporaine pour un style « rétro » destiné à remplacer « une réalité déjà nostalgique » - nostalgique peut-être d'un temps récent où la crise du pétrole n'avait pas encore remis en cause ces idées de progrès et de croissance qui accompagnent les États-nations depuis le $19^{\mathrm{e}}$ siècle. L'artiste décrit ce style « rétro » comme « une caricature de ce romantisme historique qui s'est finalement englouti dans un processus de destruction ». Il ajoute : « Mais la rumeur de ce mouvement bourgeois plus que national se chante encore comme la Lorelei et garde un étrange pouvoir. » Broodthaers, dans cette Lorelei où alternent cartes postales anciennes du Rhin et extraits de pages consacrées à la Bourse issues de différents journaux, suggère l'actualité transnationale du nationalisme romantique bourgeois et de ses « traditions inventées » dans l'Europe capitaliste des années I970. L'artiste ne manque pas de souligner le rôle clé joué par les médias de masse dans la production de ce présent « déjà nostalgique $e^{79}$ ». Brassinne se situe bien sûr quelque part dans cette configuration, qu'il contribue activement à engendrer dans la période d'après-guerre. Broodthaers cherche, lui, comme à son habitude, à marquer sa distance par l'ironie et par l'humour, notamment en s'imaginant devenir le poète romantique Clemens Brentano, depuis longtemps disparu, et en se proposant d'« éditer un nouveau Wunderhorn ${ }^{80} »$ adapté aux temps modernes. On peut supposer que cette entreprise pourrait être fort lucrative. Avec ce En lisant la Lorelei, l'artiste belge éclaire finalement ce qui empêche fondamentalement toute reconstitution : notre imaginaire historique post-romantique reste accroché à un passé produit pour les besoins de la cause nationale et commerciale. Plutôt que de s'en autoriser, l'artiste préfère le désamorcer par l'allégorie, en assumant donc de rester au niveau d'une « proposition [...] fragmentaire ${ }^{8 \mathrm{I}} \gg-$ et, de fait, cette fragmentation

${ }^{78}$ Michel Foucault, «Anti-Retro », Cabiers du Cinéma, n²5I-252, juillet-août 1974, republié dans Dits et écrits, édition établie sous la direction de Daniel Defert et François Ewald, Paris, Éditions Gallimard, 1994, t. II «1970-1975», p. 646-660.

79 Pour l'ensemble des citations et allusions à ce projet de préface (1975), voir Broodthaers, 1997, p. 89.

8o Ibid., p. 68. Il s'agit d'un autre des projets de préface. Achim von Arnim et Clemens Brentano publient entre 1805 et 1808 un très influent recueil de «chansons populaires » allemandes, Des Knaben Wunder Horn. L'ouvrage est un acteur clé de ce nationalisme romantique européen qui fait des formes populaires l'expression profonde des âmes nationales.

${ }^{81}$ Ibid., p. 92. 
est un antidote au fantasme de la reconstitution historique. Les allégories de Broodthaers, même si elles s'appuient largement sur l'appropriation d'objets historiques, ne sont pas de purs et simples « refaires »: en réinscrivant et en assemblant des items historiquement chargés, et souvent assez peu modifiés, dans un temps exogène, Broodthaers essaie de dénaturaliser certains récits et images (Waterloo, par exemple). Il tente par là même, au gré de ses projets d'exposition et d'édition, d'écrire l'histoire critique et non systématique d'un certain rapport au passé qui émergerait pendant le moment romantique en Allemagne, en France et en Grande Bretagne. Le jeu broodthaersien avec l'appropriation et la fragmentation est évidemment bien loin du modèle d'histoire appropriée porté et produit par Brassinne, dont la fascination pour la reconstitution à l'« échelle I » a aujourd'hui encore de beaux jours devant elle, alors même que la globalisation a complexifié les mécanismes de production des identités nationales et locales - récits nationaux et mémoires collectives locales étant désormais inscrits dans des réseaux d'échanges et de médiatisation globalisants, déterminant de nouveaux rapports émotionnels à l'ici et maintenant ainsi que des possibilités inédites de délocalisation des imaginaires ${ }^{82}$. Brassinne, grand bricoleur de monuments et de rituels, ne verra que les prémisses de cette nouvelle donne, puisque sa carrière se termine en 1988, à l'aube de l'institutionnalisation de la reconstitution historique, et peu de temps avant la bascule dans le monde global qui émergera après la chute du Mur de Berlin. Mais il joue certainement un rôle clé dans l'avènement de la reconstitution historique à Waterloo, dans l'invention d'une certaine relation au lieu et aussi, plus généralement, dans la valorisation de l'expérience individuelle et non spécialiste de l'Histoire — nombre de reconstituteurs de Waterloo s'étant réclamés de lui. Après les années 1970 où sa présence dans les médias décline largement et où la mémoire héroïque de la France résistante commence à se fissurer ${ }^{83}$, il finit néanmoins par se voir accorder une reconnaissance secrète à la toute fin de sa vie: celle du cinéma expérimental belge. André Colinet lui consacre son premier court métrage, sobrement intitulé Waterloo(1985), reprenant en main le projet avorté d'un réalisateur tchécoslovaque auquel Brassinne avait tout simplement demandé de lui consacrer un film ${ }^{84}$. Boris Lehman, lui, clôture sa fresque autobiographique

${ }^{82}$ Voir par exemple le traitement de l'appropriation de la culture de masse globale (le film Braveheart, Gibson, 1995) dans le discours nationaliste écossais contemporain dans Tim Edensor, 2002, p. 139-170.

$8_{3}$ Il suffira ici de citer le grand documentaire de Marcel Ophuls, Le chagrin et la pitié, 1971.

${ }^{84}$ Nous remercions André Colinet, à qui nous devons ces précisions. 
Babel (1983-199I), à Waterloo, avec le grognard moderne. La séquence se passe à l'époque de la première grande reconstitution de la bataille, en 1987. Le cinéaste, railleur devant cette image d'Épinal démesurée, y décèle le syndrome du cinéma contemporain : toujours chercher la même image ${ }^{85}$.

\section{REMERCIEMENTS}

Remerciements à mes interlocuteurs de l'INA Strasbourg/Paris et de la SONUMA; à Vincent Fannoy, ayant-droit des archives Belgavox; à Véronique Denis, échevine des sports, du tourisme et des jumelages à Braine-l'Alleud; à André Colinet, réalisateur; et à Boris Lehman, réalisateur.

8s Babel. Lettre à mes amis restés en Belgique (Lehman, 1991). Colinet, cité ci-dessus, a collaboré à de nombreuses reprises avec Lehman, notamment sur Babel. 


\title{
Reconstituer Waterloo dans les années 1960 : l'histoire selon Marcel Broodthaers et Norbert Brassinne
}

\author{
Nicolas Fourgeaud, Haute École des arts du RHIN
}

\section{RÉSUMÉ}

Avec cet article, on souhaiterait faire le portrait (contextuel, institutionnel, médiatique) de deux acteurs fondamentalement différents, mais qui font de la reconstitution historique un enjeu central de leurs pratiques dès la fin des années 1960 et la rapportent, dans un cas comme dans l'autre, à un même lieu, le champ de bataille de Waterloo, en Belgique francophone: l'artiste bruxellois Marcel Broodthaers (1924-1976) et le commerçant/reconstituteur wallon Norbert Brassine (1907-1988). En plus d'analyser quel rapport à l'histoire et à la reconstitution développent ces deux non-historiens très distincts à travers leurs actions, leurs productions ou leurs apparitions médiatiques, cet article vise aussi à revenir sur une période où la reconstitution historique en était à ses balbutiements à Waterloo, la fin des années 1960. Ce double portrait se propose donc comme une archéologie très localisée des usages et représentations de la reconstitution historique.

\section{ABSTRACT}

With this paper, the author puts forward a picture of two very different social actors who, towards the end of the 1960 in French-speaking Belgium, shared a common interest in historical reenactment at the Battlefield of Waterloo: the Brusselian artist Marcel Broodthaers (1924-1976), and the Walloon hotel manager and historical reenactor Norbert Brassinne (1907-1988). This paper analyzes how these two very different non-historians dealt with history and its reconstitution, bearing in mind their particular contexts, actions, and productions. In addition, this study provides an occasion to focus on a period when historical reconstitution in Waterloo was still in its infancy. In turn, this study proposes a localised archeology of the uses and representations of historical reconstitution.

\section{NOTE BIOGRAPHIQUE}

Nicolas Fourgeaud est enseignant en histoire de l'art à la Haute école des arts du Rhin, à Strasbourg. Ses publications récentes sont : « "Performance in Postmodern Culture", 1976: enjeux et réceptions », Marges, n²4, printemps/été 2017; «De quelques usages récents de la littérature par la performance : Benjamin Seror, Louise Hervé et Chloé Maillet », dans La tentation littéraire de l'art contemporain, Dijon, 
Les Presses du Réel, 2017, p. 267-291; « De Marina Abramović à Philip Auslander : reprise, répétition et impasses dans deux théories contemporaines sur la performance $\gg$, Marges, $\mathrm{n}^{\circ}$ 17, octobre 2013 . 